\begin{tabular}{|c|l|}
\hline Title & Luminescence of a Cooper Pair \\
\hline Author(s) & A sano, Y asuhiro; Suemune, Ikuo; Takay anagi, Hideaki; Hanamura, Eiichi \\
\hline Citation & $\begin{array}{l}\text { Physical Review Letters, 103(18), 187001 } \\
\text { https://doi.org/10.1103/PhysRevLet.103.187001 }\end{array}$ \\
\hline Issue Date & 2009-10 \\
\hline Doc URL & http://hdl.handle.net/2115/45362 \\
\hline Rights & ○ 2009 The A merican Physical Society \\
\hline Type & article \\
\hline File Information & 289_suemune.pdf \\
\hline
\end{tabular}

Instructions for use 


\title{
Luminescence of a Cooper Pair
}

\author{
Yasuhiro Asano, ${ }^{1,2}$ Ikuo Suemune, ${ }^{3,4}$ Hideaki Takayanagi, ${ }^{4,5,6}$ and Eiichi Hanamura ${ }^{7}$ \\ ${ }^{1}$ Department of Applied Physics, Hokkaido University, Sapporo 060-8628, Japan \\ ${ }^{2}$ Center of Education \& Research for Topological Science \& Technology, Hokkaido University, Sapporo 060-8628, Japan \\ ${ }^{3}$ Research Institute for Electronic Science, Hokkaido University, Sapporo 001-0021, Japan \\ ${ }^{4}$ CREST, Japan Science and Technology Agency, Kawaguchi 332-0012, Japan \\ ${ }^{5}$ Department of Applied Physics, Tokyo University of Science, Tokyo 162-8601, Japan \\ ${ }^{6}$ International Center for Nanoarichitectonics, NIMS, Tsukuba 305-0044, Japan \\ ${ }^{7}$ Japan Science and Technology Agency, Kawaguchi 332-0012, Japan
}

(Received 6 May 2009; published 30 October 2009)

This Letter theoretically discusses the photon emission spectra of a superconducting $p-n$ junction. On the basis of the second order perturbation theory for electron-photon interaction, we show that the recombination of a Cooper pair with two $p$-type carriers causes enhancement of the luminescence intensity. The calculated results of photon emission spectra explain characteristic features of observed signal in an recent experiment. Our results indicate high functionalities of superconducting light-emitting devices.

Light-emitting diodes (LEDs) usually fabricated on semiconductors have been an important element of modern technologies. Recent researches seem to focus on producing a well controlled photon and an entangled photon pair $[1,2]$ for realizing quantum information. Superconducting devices have a great advantage in producing entangled quantum states because of its coherent nature [3-5]. Superconducting LEDs [6] have been originally proposed in the context of superradiation. Today, they are a promising source of an entangled photon pair [7]. A recent theoretical study predicts the Josephson radiation in a superconducting $p-n$ junction [8]. Thus hybrids of superconductor and semiconductor LED undoubtedly have a possibility to provide a key technology in the next generation.

The radiative recombination of Cooper pairs has been observed recently in a InGaAs/InP $p$ - $n$ junction attaching onto a superconductor $\mathrm{Nb}$ [9]. The electroluminescence becomes drastically large at low temperatures below the superconducting transition temperature $T_{c}$ of Nb electrode. Surprisingly the degree of enhancement in the luminescence intensity is 1 order of magnitude. Although the experiment has shown clearly effects of superconductivity on the radiative recombination, the mechanism has been an open question. This Letter theoretically addresses this issue. We study the emission spectra of photon in a superconducting $p-n$ junction by using the second order perturbation expansion for electron-photon interaction. In the second order, we find that a peculiar recombination process to superconductivity enhances the luminescence intensity. In that recombination process, two electrons recombine with two $p$-type carriers as a Cooper pair. The theoretical results explain characteristic features of the experimental findings [9]. This Letter not only figures out a mechanism of the large luminescence intensity but also gives a guide for designing highly functional superconducting lightemitting devices.

Let us consider a $p$-type semiconductor-superconductor junction under the applied bias voltage $e V_{s d}$ as shown in Fig. 1(a). The energy is measured from the horizontal line indicated by " 0 ". The sign of energy in a $p$-type semiconductor is chosen to be opposite to that in a superconductor. We assume that a semiconductor and a superconductor are in their local equilibrium which are characterized by the local chemical potential $\mu_{p}$ and $\mu_{n}$, respectively. The edges of the conduction and valence bands are $E_{c}$ and $E_{v}$, respectively. In what follows, we use a unit of $\hbar=$ $k_{B}=c=1$, where $k_{B}$ is the Boltzmann constant and $c$ is

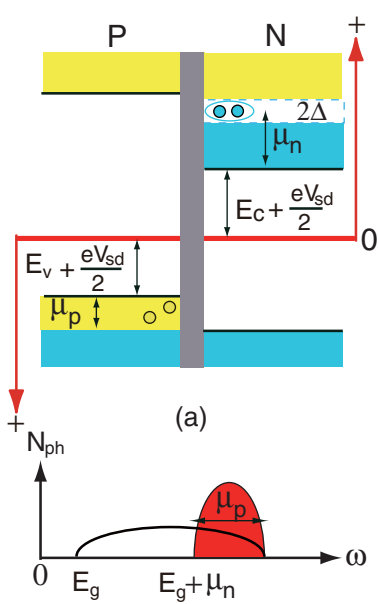

(b)

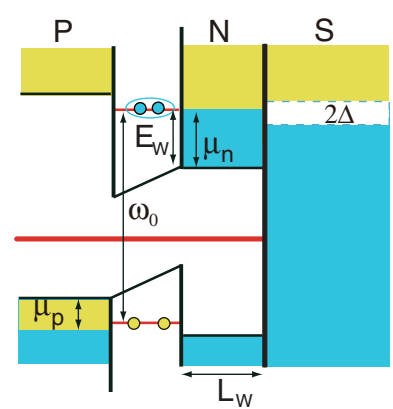

(c)

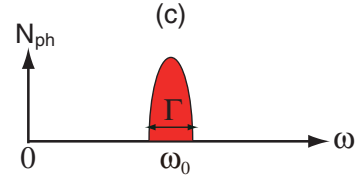

(d)
FIG. 1 (color online). Schematic energy diagram of forward biased $p$ - $n$ junctions. A theoretical model used for calculation is shown in (a). In (c), a realistic junction in experiments is illustrated. Predicted results of the photon spectra are shown in (b) and (d). 
the speed of light. The $p$-type semiconductor is described by

$$
H_{p}=\sum_{k, \sigma} \epsilon_{p}(k) b_{k, \sigma}^{\dagger} b_{k, \sigma},
$$

where $\epsilon_{p}(k)=k^{2} /\left(2 m_{p}\right)+E_{v}+e V_{s d} / 2, m_{p}$ is the effective mass, and $b_{\boldsymbol{k}, \sigma}^{\dagger}\left(b_{\boldsymbol{k}, \sigma}\right)$ is the creation (annihilation) operator of a $p$-type carrier with a wave number $\boldsymbol{k}$ and spin $\sigma=\uparrow$ or $\downarrow$. The photon states are described by

$$
H_{\mathrm{ph}}=\sum_{q} \omega_{q}\left(a_{q}^{\dagger} a_{q}+\frac{1}{2}\right)
$$

where $a_{\boldsymbol{q}}^{\dagger}\left(a_{\boldsymbol{q}}\right)$ is the creation (annihilation) operator of a photon with a wave number $\boldsymbol{q}$ and an energy $\omega_{q}$. The normal state in a metal is described by

$$
H_{n n}=\sum_{k, \sigma}\left(\frac{k^{2}}{2 m_{n}}+E_{c}+\frac{e V_{s d}}{2}\right) c_{k, \sigma}^{\dagger} c_{k, \sigma},
$$

where $c_{k, \sigma}^{\dagger}\left(c_{k, \sigma}\right)$ is the creation (annihilation) operator of an $n$-type carrier and $m_{n}$ is the effective mass. The electron-photon interaction Hamiltonian in the dipole approximation is given by

$$
H_{I}=\sum_{k, q, \sigma} B_{k, q} b_{k-q, \sigma} c_{k, \sigma} a_{q}^{\dagger}+\text { H.c., }
$$

where $B_{k, q}$ is the coupling energy. On the basis of the second order perturbation theory, the number of emitting photon $N_{\mathrm{ph}}=\sum_{q} a_{q}^{\dagger} a_{q}$ is calculated as

$$
\begin{gathered}
\left\langle N_{\mathrm{ph}}\right\rangle=\left\langle N_{\mathrm{ph}}(1)\right\rangle+\left\langle N_{\mathrm{ph}}(2)\right\rangle \\
\left\langle N_{\mathrm{ph}}(1)\right\rangle=\int_{-\infty}^{t} d t_{1} \int_{-\infty}^{t} d t_{2}\left\langle\chi_{0}\left|H_{I}\left(t_{1}\right) N_{\mathrm{ph}} H_{I}\left(t_{2}\right)\right| \chi_{0}\right\rangle \\
\left\langle N_{\mathrm{ph}}(2)\right\rangle=\int_{-\infty}^{t_{2}} d t_{1} \int_{-\infty}^{t} d t_{2} \int_{-\infty}^{t} d t_{3} \int_{-\infty}^{t_{3}} d t_{4} I(2) \\
I(2)=\left\langle\chi_{0}\left|H_{I}\left(t_{1}\right) H_{I}\left(t_{2}\right) N_{\mathrm{ph}} H_{I}\left(t_{3}\right) H_{I}\left(t_{4}\right)\right| \chi_{0}\right\rangle \\
\left|\chi_{0}\right\rangle \rightarrow|0\rangle \otimes|N\rangle \otimes|P\rangle
\end{gathered}
$$

where $|0\rangle$ is the zero photon state.

The BCS theory describes superconducting states,

$$
H_{n s}=\sum_{k, \sigma} E_{k} \gamma_{k, \sigma}^{\dagger} \gamma_{k, \sigma},
$$

where $E_{k}=\sqrt{\xi_{n}^{2}(k)+\Delta^{2}}, \xi_{n}(k)=k^{2} / 2 m_{n}-\mu_{n}, \Delta$ is the pair potential, and $\gamma_{k, \sigma}^{\dagger}\left(\gamma_{k, \sigma}\right)$ is the creation (annihilation) operator of a Bogoliubov quasiparticle. We try to consider effects of superconductivity through the Bogoliubov transformation [10]. The description in Eq. (10), however, is valid within a small energy scale near the Fermi level which is at $\tilde{\mu}_{n}=E_{c}+e V_{s d} / 2+\mu_{n}$ measured from 0 .
To apply the BCS theory to the present issue, a rule is necessary to describe the operator in the interaction picture. The transformation connects an electron operator and Bogoliubov operators by

$$
c_{\boldsymbol{k}, \sigma}^{\dagger}(t)=e^{i \tilde{\mu}_{n} t}\left(u_{k} e^{i E_{k} t} \gamma_{\boldsymbol{k}, \sigma}^{\dagger}-s_{\sigma} \boldsymbol{v}_{k} e^{-i E_{k} t} \gamma_{-\boldsymbol{k}, \bar{\sigma}}\right),
$$

in $\left\langle\chi_{0}|\cdots| \chi_{0}\right\rangle$, where $u_{k}\left(v_{k}\right)=\left\{\left[1+(-1) \xi_{n}(k) / E_{k}\right] / 2\right\}^{1 / 2}$, $s_{\sigma}=1(-1)$ for $\sigma=\uparrow(\downarrow)$, and $\bar{\sigma}$ means the opposite spin to $\sigma$. The thermal average of operators is carried out in the local equilibrium. In a $p$-type semiconductor, for instance, the average of operators is calculated in

$$
H_{p}^{\prime}=\sum_{k, \sigma} \xi_{p}(k) b_{k, \sigma}^{\dagger} b_{k, \sigma},
$$

instead of Eq. (1) with $\xi_{p}(k)=k^{2} / 2 m_{p}-\mu_{p}$. In a superconductor, the average of the Bogoliubov operators is calculated in Eq. (10). In Eq. (9), $|P\rangle$ means the state vector of $p$-type carrier in the local equilibrium and $|N\rangle$ indicates the BCS state in the local equilibrium.

The time average of the photon number $\overline{\left\langle N_{\mathrm{ph}}\right\rangle}$ corresponds to the luminescence intensity and it in the first order perturbation expansion results in

$$
\begin{aligned}
\overline{\left\langle N_{\mathrm{ph}}(1)\right\rangle}= & 2 \pi \sum_{k, \boldsymbol{q}, \boldsymbol{\sigma}}\left|B_{\boldsymbol{k}, \boldsymbol{q}}\right|^{2} f_{\boldsymbol{k}-\boldsymbol{q}}^{p}\left[u_{k}^{2} f_{k}^{n} \delta\left(\tilde{\omega}-E_{k}\right)\right. \\
& \left.+v_{k}^{2}\left(1-f_{k}^{n}\right) \delta\left(\tilde{\omega}+E_{k}\right)\right]
\end{aligned}
$$

where $\tilde{\omega}=\omega_{q}-E_{g}-\mu_{n}-\mu_{p}-\xi_{p}(\boldsymbol{k}-\boldsymbol{q}), \quad E_{g}=$ $E_{v}+E_{c}+e V_{s d}, f_{k}^{n}=\left[1-\tanh \left(E_{k} / 2 T\right)\right] / 2$, and $f_{k}^{p}=$ $\left\{1-\tanh \left[\xi_{p}(k) / 2 T\right]\right\} / 2$. This result recovers the photon spectra in a normal $p-n$ junction by tuning $\Delta \rightarrow 0$, which means that $E_{k} \rightarrow-\xi_{n}(k), u_{k} \rightarrow 0, v_{k} \rightarrow 1$ for $k<k_{F}$ and $E_{k} \rightarrow \xi_{n}(k), u_{k} \rightarrow 1, v_{k} \rightarrow 0$ for $k>k_{F}$ with $k_{F}$ being the Fermi wave number satisfying $k_{F}^{2} / 2 m_{n}=\mu_{n}$. The threshold of spectra is $E_{g}$ and the width of spectra is given by $\mu_{n}+\mu_{p}$. The spectra in Eq. (13) have a broad profile reflecting the quasiparticle density of states as schematically shown in Fig. 1(b). Equation (13) depends on temperature through the Fermi distribution function and the pair potential. We have, however, numerically confirmed that the dependence is very weak and that Eq. (13) is almost constant below and near above $T_{c}$. The general features of spectra are determined by the energy scales such as $E_{g}, \mu_{n}$, and $\mu_{n}$. They are much larger than $\Delta$.

The results of the second order perturbation are given by

$$
\begin{aligned}
I(2)= & \sum_{\boldsymbol{k}_{1} \cdots \boldsymbol{k}_{4}, \boldsymbol{q}_{1} \cdots \boldsymbol{q}_{4}, \sigma_{1} \cdots \sigma_{4}} e^{-i \Omega_{1} t_{1}-i \Omega_{2} t_{2}+i \Omega_{3} t_{3}+i \Omega_{4} t_{4}} \\
& \times B_{\boldsymbol{k}_{1}, \boldsymbol{q}_{1}}^{*} B_{\boldsymbol{k}_{2}, \boldsymbol{q}_{2}}^{*} B_{\boldsymbol{k}_{3}, \boldsymbol{q}_{3}} B_{\boldsymbol{k}_{4}, \boldsymbol{q}_{4}} Q_{P h} Q_{P} Q_{N},
\end{aligned}
$$

with $\Omega_{j}\left(\boldsymbol{k}_{j}, \boldsymbol{q}_{j}\right)=\omega_{q_{j}}-\boldsymbol{\epsilon}_{P}\left(\boldsymbol{k}_{j}-\boldsymbol{q}_{j}\right)-\tilde{\mu}_{n}$. The average of the operators $Q_{P h}, Q_{P}$, and $Q_{N}$ are calculated as follows,

$$
\begin{aligned}
Q_{P h} & =\sum_{\boldsymbol{q}_{5}}\left\langle 0\left|a_{\boldsymbol{q}_{1}} a_{\boldsymbol{q}_{2}} a_{\boldsymbol{q}_{5}}^{\dagger} a_{\boldsymbol{q}_{5}} a_{\boldsymbol{q}_{3}}^{\dagger} a_{\boldsymbol{q}_{4}}^{\dagger}\right| 0\right\rangle, \\
& =2\left(\delta_{14}^{q} \delta_{23}^{q}+\delta_{13}^{q} \delta_{24}^{q}\right),
\end{aligned}
$$




$$
\begin{aligned}
Q_{P} & =\left\langle P\left|b_{\boldsymbol{p}, \sigma_{1} 1}^{\dagger} b_{\boldsymbol{p}_{2}, \sigma_{2}}^{\dagger} b_{\boldsymbol{p}_{3}, \sigma_{3}} b_{\boldsymbol{p}_{4}, \sigma_{4}}\right| P\right\rangle, \\
& =f_{\boldsymbol{p}_{1}}^{p} f_{\boldsymbol{p}_{2}}^{p}\left(\delta_{14}^{\sigma} \delta_{23}^{\sigma} \delta_{14}^{p} \delta_{23}^{p}-\delta_{13}^{\sigma} \delta_{24}^{\sigma} \delta_{13}^{p} \delta_{24}^{p}\right),
\end{aligned}
$$

where $\delta_{i j}^{q}=\delta_{q_{i}, q_{j}}, \delta_{i j}^{\sigma}=\delta_{\sigma_{i}, \sigma_{j}}, \delta_{i j}^{p}=\delta_{p_{i}, p_{j}}$, and $\boldsymbol{p}_{j}=$ $\boldsymbol{k}_{j}-\boldsymbol{q}_{j}$. By applying the Bogoliubov transformation, we find,

$$
\begin{aligned}
Q_{N}= & \langle N|\left(u_{k_{1}} e^{i E_{k_{1}} t_{1}} \gamma_{k_{1}, \sigma_{1}}^{\dagger}-\sigma_{1} v_{k_{1}} e^{-i E_{k_{1}} t_{1}} \gamma_{-k_{1}, \bar{\sigma}_{1}}\right) \\
& \times\left(u_{k_{2}} e^{i E_{k_{2}} t_{2}} \gamma_{\boldsymbol{k}_{2}, \sigma_{2}}^{\dagger}-\sigma_{2} v_{k_{2}} e^{-i E_{k_{2}} t_{2}} \gamma_{-\boldsymbol{k}_{2}, \bar{\sigma}_{2}}\right) \\
& \times\left(u_{k_{3}} e^{-i E_{k_{3}} t_{3}} \gamma_{k_{3}, \sigma_{3}}-\sigma_{3} v_{k_{3}} e^{i E_{k_{3}} t_{3}} \gamma_{-k_{3}, \bar{\sigma}_{3}}^{\dagger}\right) \\
& \times\left(u_{k_{4}} e^{-i E_{k_{4}} t_{4}} \gamma_{k_{4}, \sigma_{4}}-\sigma_{4} v_{k_{4}} e^{i E_{k_{4}} t_{4}} \gamma_{-k_{4}, \bar{\sigma}_{4}}^{\dagger}\right)|N\rangle,
\end{aligned}
$$

which gives 12 terms. In what follows, we extract the most dominant contribution in Eq. (17). The average of $Q_{N}$ includes the following four terms

$$
\begin{aligned}
Q_{N}(S)= & u_{k_{1}} v_{k_{1}} u_{k_{3}} v_{k_{3}} \delta_{\sigma_{1}, \bar{\sigma}_{2}} \delta_{\sigma_{3}, \bar{\sigma}_{4}} \delta_{\boldsymbol{k}_{1},-\boldsymbol{k}_{2}} \\
& \times \delta_{k_{3},-k_{4}} \sigma_{1} \sigma_{3}\left[e^{i E_{k_{1}}\left(t_{1}-t_{2}\right)} e^{-i E_{k_{3}}\left(t_{3}-t_{4}\right)} f_{k_{1}}^{n}\left(1-f_{k_{3}}^{n}\right)\right. \\
& +e^{-i E_{k_{1}}\left(t_{1}-t_{2}\right)} e^{i E_{k_{3}}\left(t_{3}-t_{4}\right)} f_{k_{3}}^{n}\left(1-f_{k_{1}}^{n}\right) \\
& -e^{-i E_{k_{1}}\left(t_{1}-t_{2}\right)} e^{-i E_{k_{3}}\left(t_{3}-t_{4}\right)}\left(1-f_{k_{1}}^{n}\right)\left(1-f_{k_{3}}^{n}\right) \\
& \left.-e^{i E_{k_{1}}\left(t_{1}-t_{2}\right)} e^{i E_{k_{3}}\left(t_{3}-t_{4}\right)} f_{k_{1}}^{n} f_{k_{3}}^{n}\right]
\end{aligned}
$$

Equation (18) describes effects of superconductivity on the emission spectra because $\delta_{\sigma_{3}, \bar{\sigma}_{4}} \delta_{\boldsymbol{k}_{3},-\boldsymbol{k}_{4}}$ means the destruction of two electrons as a Cooper pair. A recombination process in $Q_{N}(S)$ is schematically illustrated in Fig. 2(a). The remaining eight terms in $Q_{N}$ describe the emitting processes shown in Fig. 2(b) and give the luminescence intensity proportional to $\left.\overline{\left\langle N_{\mathrm{ph}}(1)\right.}\right\rangle^{2}$. We will show that $Q_{N}(S)$ gives large contribution to the emission spectra at $\delta \boldsymbol{q}=\boldsymbol{q}_{1}+\boldsymbol{q}_{2}=0\left(\delta \boldsymbol{q}=\boldsymbol{q}_{3}+\boldsymbol{q}_{4}=0\right.$ in other words $)$

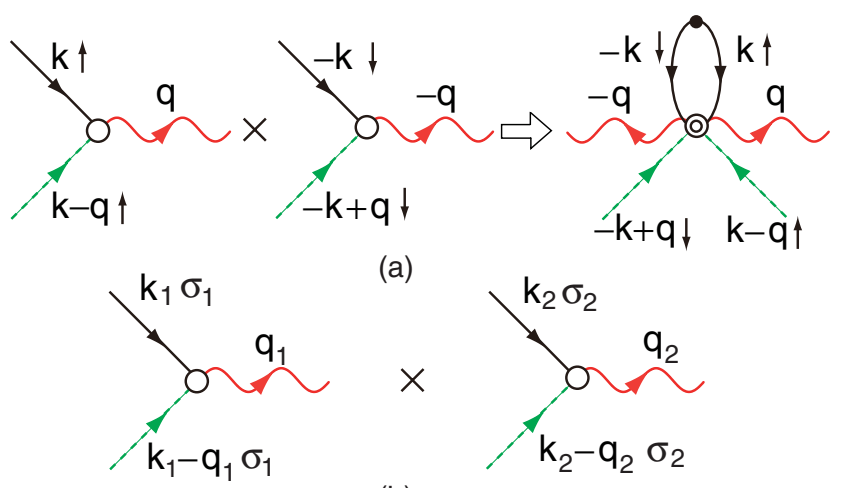

(b)

FIG. 2 (color online). Recombination processes in the second order perturbation expansion, where solid, broken, and wavy lines represent the propagation of an electron, a $p$-type carrier, and a photon, respectively. In (a), a recombination of a Cooper pair in $Q_{N}(S)$ is shown. In (b), a recombination process other than $Q_{N}(S)$ is illustrated.
[11]. Substituting Eqs. (15), (16), and (18) into Eq. (14) and carrying out time integrations, we obtain

$$
\begin{gathered}
\overline{\left\langle N_{\mathrm{ph}}(2)\right\rangle}=4 \pi|B|^{4} \sum_{q, \sigma} \delta\left(\Omega_{\boldsymbol{k}_{F}, \boldsymbol{q}}\right) I_{0}, \\
I_{0}=\sum_{k}\left[\frac{f_{k}^{n}\left(1-f_{k}^{n}\right)}{\left(E_{k}-i / \tau\right)^{2}}+\frac{f_{k}^{n}\left(1-f_{k}^{n}\right)}{\left(E_{k}+i / \tau\right)^{2}}\right. \\
\left.+\frac{\left(f_{k}^{n}\right)^{2}+\left(1-f_{k}^{n}\right)^{2}}{E_{k}^{2}+(1 / \tau)^{2}}\right] \frac{\Delta^{2}}{E_{k}^{2}},
\end{gathered}
$$

where a relaxation time $\tau$ is introduced to remove effects of the perturbation at $t \rightarrow-\infty$. We neglect dependence of $B$ on wave numbers and assume $f_{\boldsymbol{k}_{F}-\boldsymbol{q}}^{p}=1$. At $1 / \tau=0$, $I_{0}=\pi N_{0} / 2 \Delta$ essentially diverges for small $\Delta$ with $N_{0}$ denoting the normal density of states in a superconductor at the Fermi energy. The singular behavior at small $\Delta$ in Eq. (20) is a sign of the large luminescence intensity due to superconductivity.

We first show mathematical reasons of the singularity. Then we will discuss the physics behind the phenomenon. A two-photon emitting process in $Q_{N}(S)$ is illustrated in Fig. 2(a). The annihilation of a Cooper pair is described by $c_{-k, \downarrow} c_{k, \uparrow}$ which includes an operator $\gamma_{k, \uparrow}^{\dagger} \gamma_{k, \uparrow}$. Let us assume that the energy of the initial state is zero. In the first order expansion, the operation of $\gamma_{k, \uparrow}$ to the BCS state decreases energy by $E_{k}+\tilde{\mu}_{n}$. At the same time, a $p$-type carrier with energy $\epsilon_{p}(\boldsymbol{k}-\boldsymbol{q})$ is destructed and a photon with energy $\omega_{q}$ is created. Thus the energy of the intermediate state $\delta E_{1} \quad$ results in $\delta E_{1}=\omega_{q}-\epsilon_{p}(\boldsymbol{k}-\boldsymbol{q})-E_{k}-\tilde{\mu}_{n}=$ $\Omega_{k, q}-E_{k}$. In the perturbation expansion, $\delta E_{1}$ becomes the energy denominator. In the second order, the operation of $\gamma_{k, \uparrow}^{\dagger}$, the destruction of a $p$-type carrier, and the creation of a photon gain energy by $E_{k}-\tilde{\mu}_{n},-\boldsymbol{\epsilon}_{p}(-\boldsymbol{k}+\boldsymbol{q})$, and $\omega_{-q}$, respectively. Therefore the difference in energy between the intermediate state and the final one becomes $\delta E_{2}=\omega_{q}-\epsilon_{p}(\boldsymbol{k}-\boldsymbol{q})+E_{k}-\tilde{\mu}_{n}=\Omega_{\boldsymbol{k}, \boldsymbol{q}}+E_{k} . \quad$ The perturbation theory requires the energy conservation between the initial state and the final one (i.e., $\delta E_{1}+\delta E_{2}=$ 0 ), which leads to $2 \Omega_{k, q}=0$. As a result, a small value of $E_{k}$ remains in the denominator as shown in Eq. (20). The physics behind the phenomena is simple. The BCS state has an ability to emit a pair of photons with remaining in its state almost unchanged because the BCS state is the eigenstate of $\gamma_{k, \uparrow}^{\dagger} \gamma_{k, \uparrow}$. The equation $\Omega_{\boldsymbol{k}_{F}, \boldsymbol{q}}=0$ describes the emitting condition of two photons. The threshold and width of spectra are $E_{g}+\mu_{n}$ and $\mu_{p}$, respectively. In Fig. 1(b), we schematically show predicted spectra in the second order process.

The singular behavior in perturbation expansion implies an importance of higher order terms for predicting the luminescence intensity quantitatively. Here we do not discuss this issue, but choose an alternative way of regularizing the obtained results. In what follows, we introduce a finite relaxation time. First we consider a mean free time 

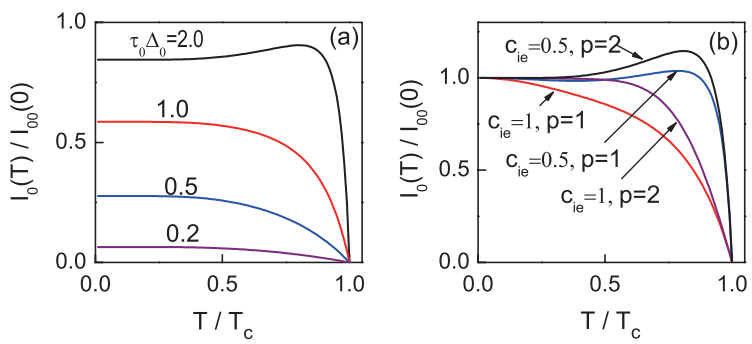

FIG. 3 (color online). Temperature dependence of luminescence intensity in Eqs. (19) and (20). In (a), we consider relaxation time due to the elastic impurity scatterings by $\alpha=\tau_{0} \Delta_{0}$. In (b), we consider the relaxation due to inelastic scatterings.

due to elastic impurity scatterings $\tau_{0}$. At $T=0$, we obtain $I_{0}=I_{00}(0) 2 \alpha^{2} /\left(\sqrt{1+\alpha^{2}}\left(\alpha+\sqrt{1+\alpha^{2}}\right)\right)$, where $\alpha=$ $\tau_{0} \Delta_{0}, \Delta_{0}$ is the pair potential at the zero temperature and $I_{00}(0)=\pi N_{0} / 2 \Delta_{0}$ is Eq. (20) at $T=0$ and $1 / \tau=0$. At $T \lesssim T_{c}$, we find

$$
\frac{I_{0}}{I_{00}(0)} \approx \begin{cases}c_{0} \alpha^{2}\left(\Delta / \Delta_{0}\right)^{2} \Delta_{0} / T & \alpha \lesssim 1, \\ \alpha^{3}\left(\Delta / \Delta_{0}\right)^{2} & \alpha \gg 1,\end{cases}
$$

where $c_{0}$ is a constant of the order of unity. In Fig. 3(a), we show $I_{0}$ as a function of temperature for several choices of $\alpha$, where we describe the dependence of $\Delta$ on temperature by the BCS theory. The amplitude of $I_{0}$ at $T=0$ is suppressed in the dirty limit as shown in a result with $\alpha=0.2$. The amplitude at $T=0$ increases with increasing $\alpha$. At $\alpha=1, I_{0}(0)$ has almost the same amplitude as $I_{00}(0)$. When we increase $\alpha$ up to 2.0, the results show a bump just below $T_{c}$. Next we consider inelastic scatterings described by $1 / \tau_{i e}=C_{i e}\left(T / T_{c}\right)^{p}$, where $C_{i e}$ is a coupling constant and $p$ depends on scattering sources such as $p=$ 1 for electron-phonon scatterings and $p=2$ for repulsive electron-electron interaction. In Fig. 3(b), we calculate $I_{0}$ for several choices of $C_{i e}$ and $p$. Since $1 / \tau_{i e} \rightarrow 0$ at $T=0$, the amplitude is close to $I_{00}(0)$ at $T=0$. When we decreases $C_{i e}$, the bump appears below $T_{c}$. For $1 / \tau \lesssim \Delta_{0}$, the luminescence intensity at $T \lesssim T_{c}$ is then given by

$$
\overline{\left\langle N_{\mathrm{ph}}(2)\right\rangle} \approx 4 \pi c_{0}|B|^{4} N_{0} \frac{(\tau \Delta)^{2}}{T} \sum_{q} \delta\left(\Omega_{k_{F}, q}\right) .
$$

Finally we modify Eq. (22) to describe the photon spectra in realistic junctions as shown in Fig. 1(c). A superconductor is attached to an $n$-type semiconductor whose thickness $L_{w}$ is about $30-50 \mathrm{~nm}$ [9]. The proximity effect enables the penetration of Cooper pairs into the $n$-type semiconductor. In experiments, photons are emitted mainly from a quantum well which is sandwiched by the $p$ and $n$-type semiconductor. The pair amplitude in the quantum well can be proportional to $\Delta e^{-L_{w} / \xi_{T}}$ with $\xi_{T}=$ $\sqrt{D / 2 \pi T}$ and $D$ being the diffusion constant in the $n$-type semiconductor. The quantum well would be replaced by a quantum dot near future. The level in the quantum well (dot) $E_{w}$ should coincide with the Fermi level in the $n$-type semiconductor $\mu_{n}$. Namely, $\mid E_{w}-$ $\mu_{n} \mid$ must be less than both the Thouless energy $E_{\mathrm{Th}}=$ $D / L_{w}^{2}$ and $\Delta$. This resonant condition is particularly important for a Cooper pair to penetrate into the quantum well (dot). The emission spectra have a peak at $\omega_{0}$ and the peak width is given by $\Gamma=t_{w}^{2} N_{0}$, where $t_{w}$ is the transfer integral between the quantum well (dot) and the semiconductor. The argument above is summarized by an equation for $T \lesssim T_{c}$

$$
\overline{\left\langle N_{\mathrm{ph}}(2)\right\rangle} \approx|B|^{4} N_{0} \Gamma \sum_{q, \sigma} \frac{\Delta^{2} \tau^{2} e^{-2 L_{W} / \xi_{T}} / T}{\left(\omega_{q}-\omega_{0}\right)^{2}+(\Gamma)^{2}},
$$

where we introduce the Lorentz resonant function by hand. In the experiment, $\xi_{T}$ estimated to be $680 \mathrm{~nm}$ at $4 \mathrm{~K}$ is much larger than $L_{w}$ below $T_{c}$. Thus the theoretical results in Fig. 3 may describe experimental results of the luminescence intensity. In fact, the experimental results of Fig. 6(b) in Ref. [9] show a very similar line shape to that in Fig. 3(a) with $\alpha=1$.

In conclusion, we have studied the photon emission spectra in a superconducting $p-n$ junction based on the second order perturbation theory for electron-photon interaction. We have found in the second order expansion that a peculiar recombination process to superconductivity enhances the luminescence intensity. The theoretical results explain temperature dependence of the luminescence intensity observed in an recent experiment.

[1] P. Michler, A. Kiraz, C. Becher, W. V. Schoenfeld, P. M. Petroff, Lidong Zhang, E. Hu, and A. Imamogulu, Science 290, 2282 (2000).

[2] O. Benson, C. Santori, M. Pelton, and Y. Yamamoto, Phys. Rev. Lett. 84, 2513 (2000).

[3] I. Chiorescu, P. Bertet, K. Semba, Y. Nakamura, C. J. P. M. Harmans, and J.E. Mooij, Nature (London) 431, 159 (2004).

[4] A. Wallraff, D. I. Schuster, A. Blais, L. Frunzio, R.-S. Huang, J. Majer, S. Kumar, S. M. Girvin, and R. J. Schoelkopf, Nature (London) 431, 162 (2004).

[5] N. Katz, M. Ansmann, R. C. Bialczak, E. Lucero, R. McDermott, M. Neeley, M. Steffen, E. M. Weig, A. N. Cleland, J. M. Martinis, and A. N. Korotkov, Science 312, 1498 (2006).

[6] E. Hanamura, Phys. Status Solidi B 234, 166 (2002).

[7] I. Suemune, T. Akazaki, K. Tanaka, M. Jo, K. Uesugi, M. Endo, H. Kumano, E. Hanamura, H. Takayanagi, M. Yamanishi, and H. Kan, Jpn. J. Appl. Phys. 45, 9264 (2006).

[8] P. Recher, Y. V. Nazarov, and L. Kouwenhoven, arXiv:0902-4468.

[9] Y. Hayashi, K. Tanaka, T. Akazaki, M. Jo, H. Kumano, and I. Suemune, Appl. Phys. Express 1, 011701 (2008).

[10] J. Unterhinninghofen, D. Manske, and A. Knorr, Phys. Rev. B 77, 180509(R) (2008).

[11] The speed of light is much larger than the Fermi velocity. In such case, this condition becomes $\left|\boldsymbol{q}_{1}\right| \sim\left|\boldsymbol{q}_{2}\right|$. 